$\begin{array}{llr}R & =\text { gas constant } & {\left[1.987 \mathrm{cal} / \mathrm{mol} \cdot{ }^{\circ} \mathrm{K}\right]} \\ T & =\text { absolute temperature } & {\left[{ }^{\circ} \mathrm{K}\right]} \\ v & =\text { liquid molar volume } & {[\mathrm{cc} / \mathrm{mol}]} \\ w_{i j} & =\text { adjustable parameter } & {[-]} \\ x_{i} & =\text { liquid-phase mole fraction of component } i & {[-]} \\ y_{i} & =\text { vapor-phase mole fraction of component } i & {[-]} \\ z & =\text { compressibility factor } & {[-]} \\ \gamma & & \\ \varphi & & \text { activity coefficient }\end{array}$

$\langle$ Subscript $\rangle$

$\begin{array}{ll}1,2, i & =\text { component } \\ \text { cal } & =\text { calculated } \\ \exp & =\text { experimental } \\ m & =\text { mixture }\end{array}$

$\langle$ Superscript〉

$s \quad=$ pure saturated

Literature Cited

1) Dymond, J. H. and E. B. Smith: "The Virial Coefficients of Gases", Clarendon Press (1969).

2) Hirayama, K. and T. Toriumi: Bulletin of The Chemical Research Institute of Non-Aqueous Solutions, Tohoku University, 10, 29 (1961).

3) Morisue, T., K. Noda and K. Ishida: J. Chem. Eng. Japan, 5, 217 (1972).

4) Morisue, T., K. Noda and K. Ishida: ibid., 6, 355 (1973).

5) Noda, K., K. Fukawa, M. Yanagisawa and K. Ishida: Kagaku Kögaku, 35, 245 (1971).

6) O'Connell, J. P., and J. M. Prausnitz: Ind. Eng. Chem., Process Design and Develop., 6, 245 (1971).

7) Renon, H. and J. M. Prausnitz: AIChE J., 14, 135 (1968).

8) Rowlinson, J. S.: "Liquids and Liquid Mixtures", Butterworth (1969).

9) Wilson, G. M.: J. Am. Chem. Soc., 86, 127 (1964).

\title{
BEHAVIOR OF SINGLE AIR BUBBLES HELD STATIONARY IN DOWNWARD FLOWS
}

\author{
EIICHI KOJIMA*1, TAKASHI AKEHATA AND TAKASHI SHIRAI \\ Research Laboratory of Resources Utilization, Tokyo Institute of \\ Technology, Tokyo, 152
}

The behavior of single air bubbles was studied by holding them stationary by downward flows. Two series of experiments, involving undisturbed flow and disturbed flow by an immersed cylinder, respectively, were conducted in the Reynolds number range 2000-5000 and at the equivalent spherical diameter of bubble $0.4-2 \mathrm{~cm}$. In the disturbed flow, the intensity of turbulence was 0.13 and the integral scale was of the same order of magnitude as bubble diameter. And although the bubble shape was changed considerably from time to time by the effect of turbulence, the relative velocity of bubbles was almost the same as in the undisturbed flow. The averaged ratio of the wake region volume to the bubble volume for the two cases was 4.7.

The motion and shape of bubbles have been studied in various aspects, and have been reported to be influenced by liquid viscosity, surface tension and the presence of a small amount of surface active agents $^{6,8,11)}$.

When we are concerned about individual bubbles in a swarm of bubbles, the effect of neighboring bubbles, especially the effect of turbulence on the motion or shape, should be taken into account. This is because they rise in a turbulent field created by neighboring bubbles. Information on bubble wakes is scarce.

The effect of turbulence on the drag coefficients of solid spheres has been studied extensively and there

Received April 26, 1974

*1 Present address for correspondence: Dept. of Biochemical Eng., Tokyo Univ. of Education, Tokyo, 152 has been a review ${ }^{4}$. But as for bubbles, the effect of turbulence has not been investigated systematically. This is partly because it is difficult to hold a bubble at a fixed position in a flow field with arbitrarily controlled turbulence characteristics.

Baker and $\mathrm{Chao}^{3)}$ photographed bubbles moving in a turbulent water flow and found that the relative velocity of bubbles differs slightly from the terminal velocity of single air bubbles in a stagnant liquid. Davidson et al..$^{5)}$ and also Moo-Young et al. ${ }^{9)}$ studied the behavior of bubbles held stationary in countercurrent flow apparatus with a flow spoiler consisting of a bundle of plastic drinking straws glued together. Moo-Young et al. found that turbulence in the continuous phase caused surprisingly little change in bubble velocities and shapes as compared with free rise situations. In the above work ${ }^{3,5,9)}$, turbulence 
characteristics such as intensity, scale and spectrum of turbulence are not mentioned.

Information on the size of wakes of single bubbles is useful to estimate the liquid flow velocity in bubble columns. But only a few data have been reported. Subramanian et $a l^{10)}$ determined the wake volumes of small gas bubbles of up to $3 \mathrm{~mm}$ at very low gas flow rate by a dye tracer technique, and found that the ratio of wake volume to bubble volume varied from 0.9 to 1.55 for $10<R e=d U / \nu<100$, remaining constant thereafter up to $R e=500$.

In this work, by using a countercurrent flow apparatus, single air bubbles were held at a fixed position in the test section. Bubble relative velocities, distortion of bubbles and volume of wakes accompanying bubbles were measured in an undisturbed and a disturbed flow. The disturbed flow was created by an immersed cylinder, and turbulence characteristics such as intensity and scale of turbulence and one-dimensional energy spectrum distribution of liquid velocity fluctuation were measured.

\section{Experimental Apparatus and Procedures}

The countercurrent flow apparatus for holding single bubbles in the test section is shown schematically in Fig. 1. Liquid is fed by the pump (4) to the top where the diameter is $15 \mathrm{~cm}$, and it flows down through honeycombs and a confined nozzle (1) where the velocity profile is allowed to develop to uniform distribution. The test section is $5 \mathrm{~cm}$ in diameter and $25 \mathrm{~cm}$ long, and is surrounded by a transparent box for avoiding the distortion of image when the bubbles are photographed. A single bubble was introduced at the bottom of the test section by a syringe and by controlling the flow rate by the cock (3), single bubbles were held stationary at a position $15 \mathrm{~cm}$ from the inlet of the test section. Liquid used was $10 \% \mathrm{NaCl}$ aqueous solution, its density and viscosity being $1.06 \mathrm{~g} / \mathrm{cm}^{3}$ and 1.10 c.p., respectively. The temperature was kept at $30.0^{\circ} \mathrm{C}$. As the water and the sodium chloride were not purified especially, this experiment is thought to be for a contaminated system.

The bubble relative velocity and wake volume were measured by taking photographs of bubbles and tracer particles flowing with the liquid, using a camera with $200 \mathrm{~mm}$ telescopic lens. The tracer particles were styrene polymer particles of 20-30 mesh and a density of 1.06. In the dark background, the tracer particles were lighted by a slit beam.

In the case of disturbed flow, a glass cylinder $1.0 \mathrm{~cm}$ in diameter was fixed at the inlet of the test section perpendicular to the flow, and measurements were carried out $15 \mathrm{~cm}$ downstream from the cylinder. In the measurements of turbulence characteristics, a

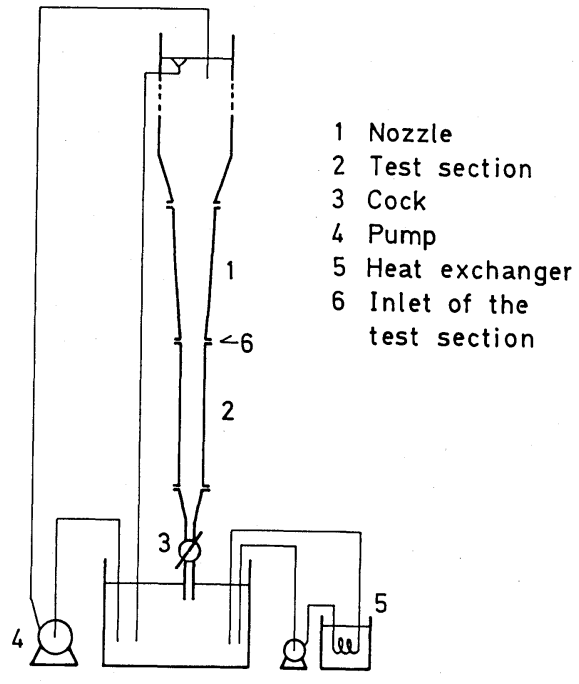

Fig. 1 Schematic of countercurrent flow apparatus

constant-temperature anemometer (Nihon Kagaku Kogyo Co., Ltd.) with a cylindrical quartz-coated hot-wire probe (Thermosystems Inc.) was used, the sensing element of which was $1.0 \mathrm{~mm}$ long and $0.051 \mathrm{~mm}$ in diameter.

\section{Experimental Results and Discussion}

\section{Relative velocity of single bubbles}

From the length of the trajectory of tracer particles on the photographs and the exposure time, the bubble relative velocity was calculated. The calculations were done for trajectories that are straight and not involved in the wake of bubbles. The equivalent spherical diameter $d$ of the bubbles was calculated on the assumption that the bubble is of spheroidal shape with length of major axis $a$ and minor axis $b$, viz,

$$
d=\sqrt[3]{a^{2} b}=\sqrt[3]{6 V_{B} / \pi}
$$

When $d$ is larger than $1.5 \mathrm{~cm}$, the value of $d$ calculated from Eq. (1) is $5 \%$ larger at the maximum as compared with the value of $d$ calculated on the assumption that the shape is a spherical cap.

In the case of undisturbed flow, and when no bubble was introduced, the radial distribution of liquid velocity at $15 \mathrm{~cm}$ from the test section inlet is shown in Fig. 2 A. It can be seen that the velocity profile is uniform over the entire cross-sectional area. At this position single bubbles could be held stationary for several minutes without controlling the liquid flow rate. Examples of photographs for undisturbed flow are shown in Figs. $3 \mathbf{A}$ and $\mathbf{B}$. The exposure time was $1 / 8 \mathrm{sec}$. The bubble relative velocity is shown in Fig. 4 as a function of equivalent diameter $d$. Open circles represent values for undisturbed flow cases. The relative velocities are almost constant at $24 \mathrm{~cm} / \mathrm{sec}$ for equivalent diameters between 0.5 and $2.0 \mathrm{~cm}$. These values are similar to 

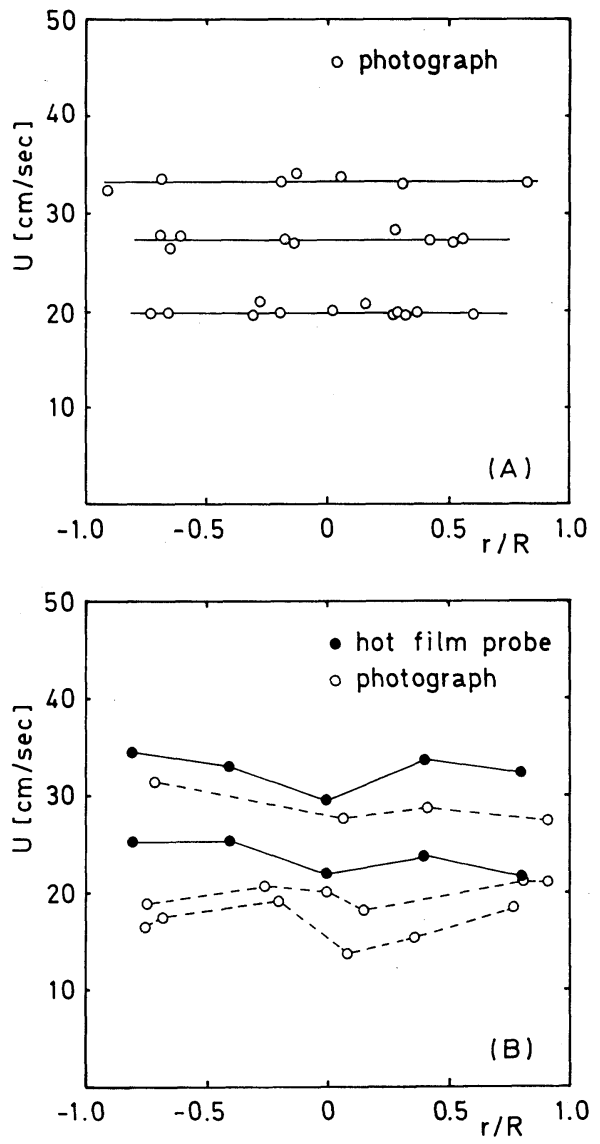

A: undisturbed, $15 \mathrm{~cm}$ from the inlet of the test section

B: disturbed, $15 \mathrm{~cm}$ from the cylinder

Fig. 2 Radial profile of liquid velocity

those in quiescent liquids ${ }^{8)}$ and a flow system ${ }^{3)}$.

For the case of disturbed flow, the radial velocity profile $15 \mathrm{~cm}$ from the cylinder is shown in Fig. 2 B.

Since the velocity downstream of the cylinder varies with time, the liquid velocity distribution measured by photographs (represented by open circles) is instantaneous at some instant. The distribution by anemometry which is represented by black circles was obtained by measurement of one minute, and the velocity in the central region is about $10 \%$ lower than in the outer region. Two kinds of distribution correspond to a sample and sample mean, respectively.

Although the liquid velocity fluctuation was large the single bubbles could be held at a fixed position by frequent and careful regulation of the cock, and breakage of bubbles was occasionally observed, especially for large bubbles. Figs. $3 \mathbf{C}$ and $\mathbf{D}$ are examples of photographs of single bubbles in disturbed flow. The bubble relative velocities for the disturbed case are plotted in Fig. 4 as black circles. These values are slightly higher than those for undisturbed flow. In almost all cases, the bubble relative velocities were calculated from the particle trajectories
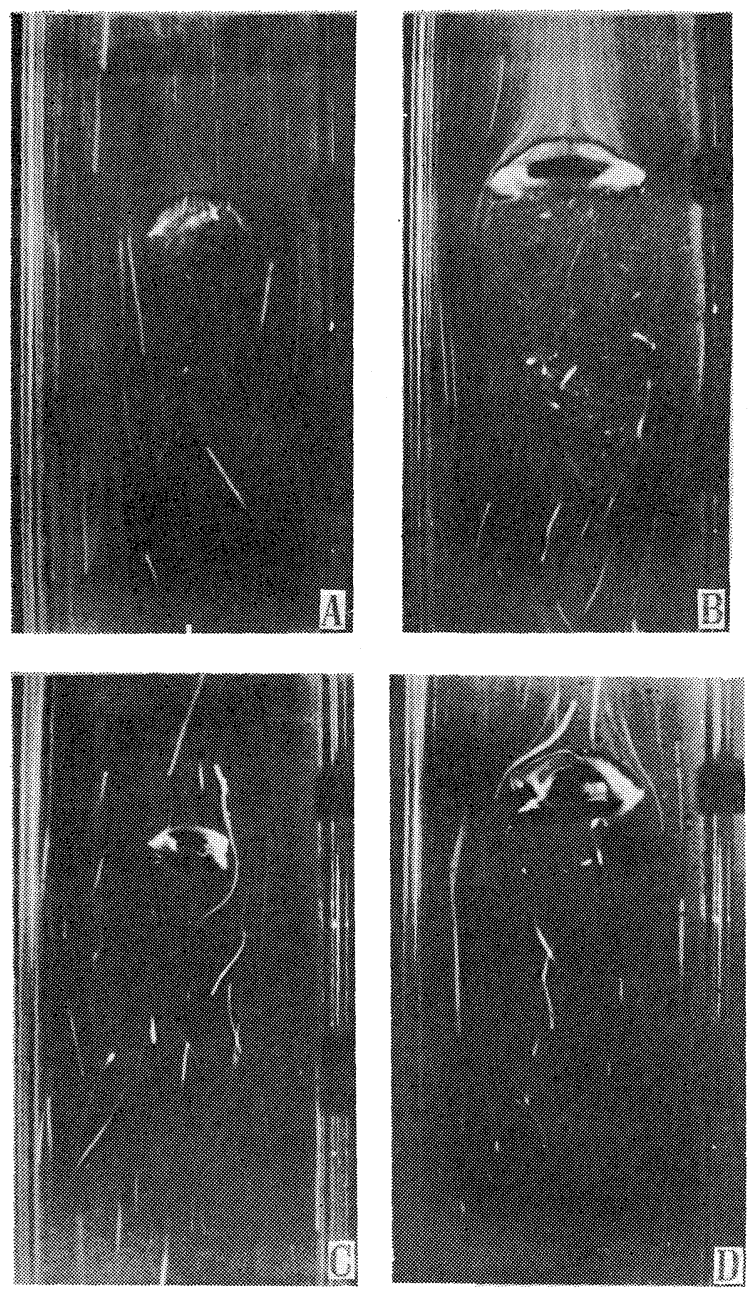

$\mathrm{A}(\boldsymbol{R e}=2700), \mathrm{B}(\operatorname{Re}=5300)$ : in the undisturbed flow $\mathrm{C}(R e=2700), \mathrm{D}(R e=5500)$ : in the disturbed flow

Fig. 3 Photographs of single air bubbles held at a fixed position

near the wall, that is, from the trajectories which are not disturbed by the wake of bubbles. Thus when the velocity profile in Fig. $2 \mathrm{~B}$ that shows the $10 \%$ lower velocity value in the central zone is taken into account, the relative velocity of single bubbles in the disturbed flow is substantially the same as that in the undisturbed case, notwithstanding the presence of the violent fluctuation of velocity.

Fig. 5 shows drag coefficient as a function of Reynolds number. Solid line represents data in quiescent liquids ${ }^{8}$. The values of drag coefficient in this study are slightly greater than those in quiescent liquids. Since the wall effect calculated as the value of $1-\left(U / U_{\infty}\right)$ is ${ }^{13)} 0.1$ and 0.2 for the equivalent diameter of $1.5 \mathrm{~cm}$ and $1.8 \mathrm{~cm}$, respectively, the slightly higher values of drag coefficient may be attributed to the wall effect.

\section{Shape of bubbles}

The ratio of the minor axis to the major axis of bubbles, deformation index $b / a$, is shown in Fig. 6 


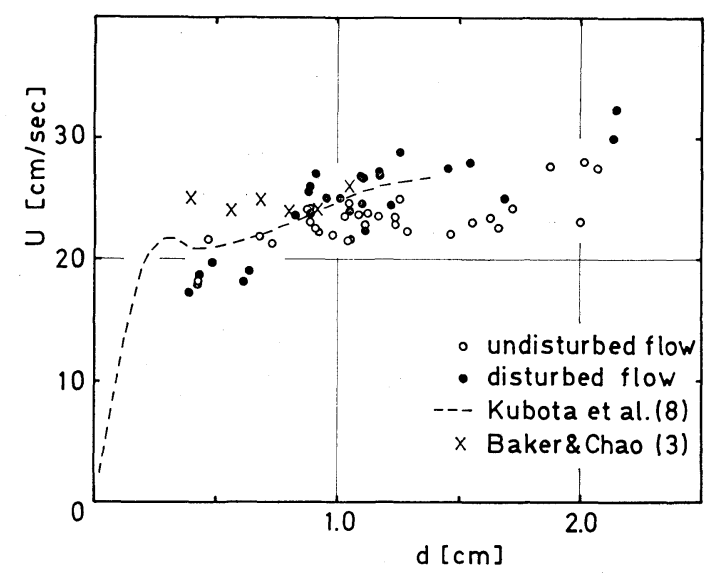

Fig. 4 Relative velocity of single air bubbles

as a function of equivalent spherical diameter $d$. Open circles represent the values of the undisturbed case. The value of $b / a$ decreases as $d$ increases, and these values are similar to those in quiescent water $^{8)}$. Black circles are for the disturbed case, where the shape of bubbles changed from time to time and occasional breakage of bubbles was observed as previously described. Thus the values of $b / a$ shown here are those at some instant. These data show larger scatter as compared with those in the non-disturbed case. It seems that the lowest value of $b / a$ is the same as for the non-disturbed case. But larger values were also obtained when a bubble, especially a large bubble, is trapped in the wake of the cylinder and is drawn upward at the top and downward at the rear edge by the effect of instantaneous liquid velocity distribution. It can be pointed out that the shape of bubbles is determined not only by liquid properties but also by the turbulence contained in the flow, i.e., change in velocity with time and position to a large extent.

\section{Volume of wake region}

In the photographs it is observed that downstream of a bubble there is a liquid accompanying the bubble. This will be called the wake region. Reynolds numbers in this study are in the range 2000 to 5000 . In this range the boundary between the wake region and the outer region is not so clear as that in the lower Reynolds number range experiments ${ }^{10,12)}$. The wake region is here determined in the photographs as a region immediately below the bubble in which the trajectory of a tracer particle is definitely disturbed and the angle between a trajectory and the main flow is almost rectangular. The volume of the wake region was calculated as the volume of a cylinder having a diameter of the length of the bubble major axis, $a$, and a height of wake length. Fig. 7 shows the ratio of the wake region volume to the bubble volume as a function of Reynolds number. The values of $V_{W} / V_{B}$ exhibit a rather large scatter, owing to the difficulty in

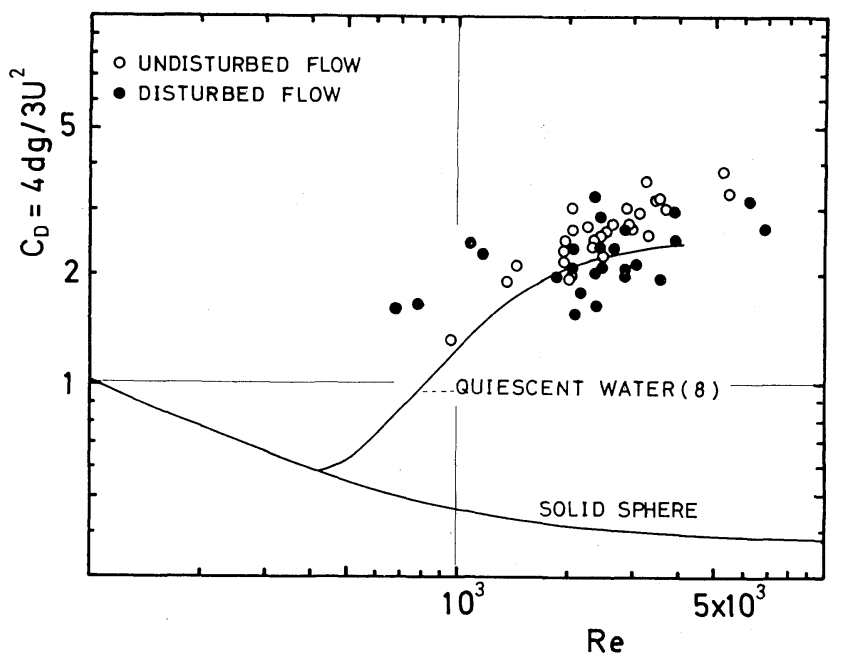

Fig. 5 Drag coefficient as a function of Reynolds number

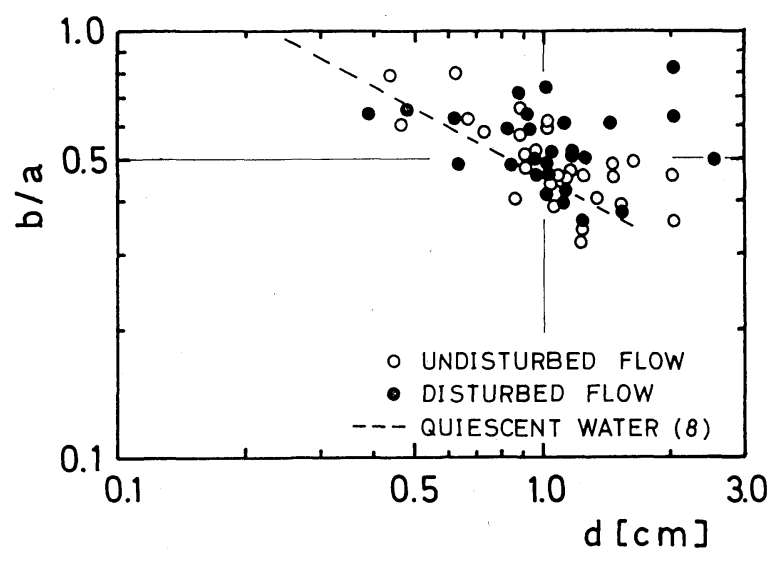

Fig. 6 Bubble deformation index as a function of equivalent diameter

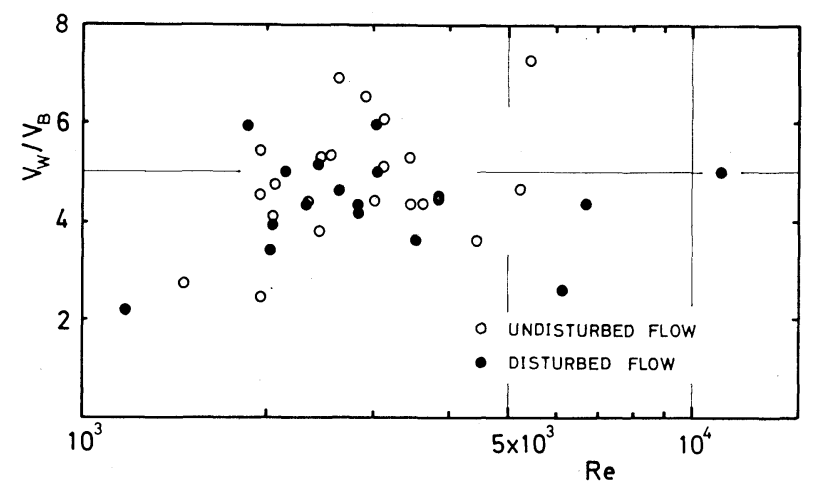

Fig. 7 Ratio of the wake volume to the bubble volume

determining the wake region boundary. It has been reported $^{1)}$ that the size of wake accompanying a cylinder decreases when turbulence exists in a flow. But from this experiment, it seems that the value of $V_{W} / V_{B}$ takes a constant value for both the undisturbed and disturbed cases. The averaged value of $V_{W} / V_{B}$ was obtained as 4.7 in the equivalent diameter range of 0.4 to $1.8 \mathrm{~cm}$. In the range of Reynolds number smaller than 500 , the values of $V_{W} / V_{B}$ of 


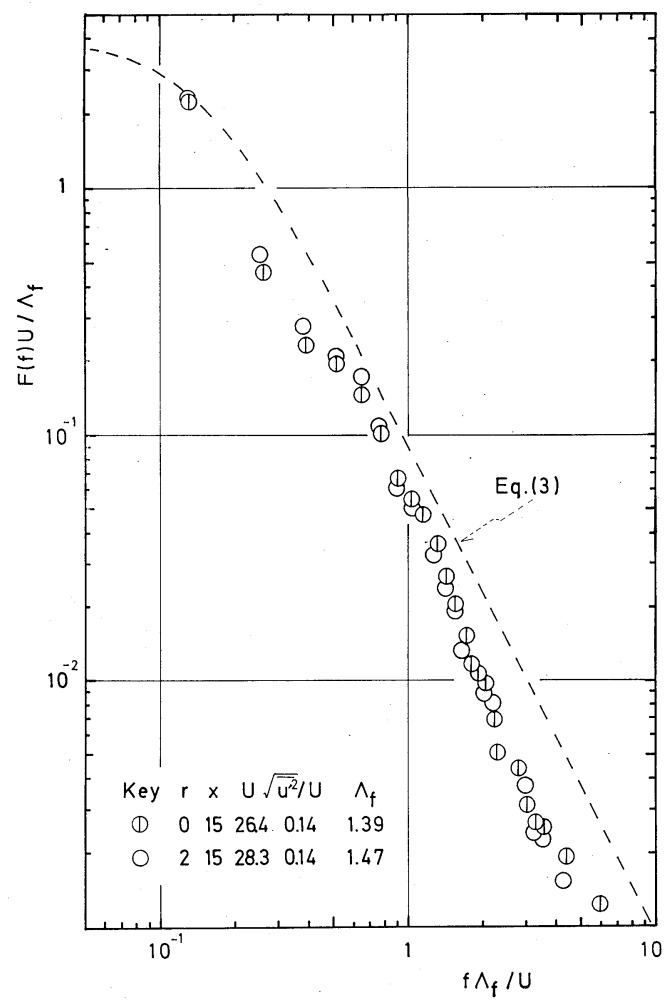

Fig. 8 One-dimensional energy spectrum distribution in the disturbed flow $(15 \mathrm{~cm}$ from the cylinder)

single bubbles take a constant value of $1.4^{10)}$. The result of this study does not agree with an estimation obtained from the extrapolation of smaller Reynolds number experiment. This is considered due to the difference in the wake structure at large Reynolds number.

\section{Turbulence characteristics of disturbed flow}

In the case of disturbed flow liquid velocity was measured by a constant-temperature anemometer. And from the digitalized signal of anemometer output, the intensity, scale and one-dimensional energy spectrum distribution of liquid velocity fluctuation were calculated. The frequency of analogue-todigital conversion was $1 \mathrm{KHz}$, and the data acquiring time was $10 \mathrm{sec}$. The method of calculation of spectrum distribution is due to Akaike ${ }^{2)}$. After the one-dimensional spectrum distribution $F(f)$ is obtained, the integral scale of turbulence $\Lambda_{f}$ was calculated as follows.

$$
\Lambda_{f}=(U / 4) F(0)
$$

Table 1 shows the turbulence characteristics. The liquid velocity of the case A corresponds to the relative velocity of bubbles in the disturbed case. The values of integral scale were about $1 \mathrm{~cm}$. This means that the magnitude of the integral scale is of the same order as the diameter of the immersed cylinder, and also of the bubbles. The intensity of turbulence was about 0.13 over the entire cross-sectional area. Using the value of $\Lambda_{f}$ and of $U$, the spectrum function $F(f)$
Table 1 Turbulence characteristics measured $15 \mathrm{~cm}$ from the immersed cylinder

\begin{tabular}{lcccc} 
& \multicolumn{3}{c}{$A$} & $B$ \\
\hline$r[\mathrm{~cm}]$ & 0 & 1 & 2 & 0 \\
\hline$U[\mathrm{~cm} / \mathrm{sec}]$ & 26.3 & 31.0 & 28.0 & 17.8 \\
$\sqrt{\overline{u^{\prime 2}}} / U$ & 0.14 & 0.11 & 0.13 & 0.13 \\
$\Lambda_{f}[\mathrm{~cm}]$ & 1.3 & 0.94 & 1.3 & 0.84 \\
\hline
\end{tabular}

was normalized and is shown in Fig. 8 for two radial positions of $r=0$ and 2. The spectrum distributions at the two positions are similar to each other and have no remarkable peak. These are relatively well correlated by the equation

$$
F(f) U / \Lambda_{f}=4 /\left\{1+\left(4 \pi^{2} f^{2} / U^{2}\right) \Lambda_{f}^{2}\right\}
$$

The difference between the equation and the data increases as the normalized frequency increases.

It is said ${ }^{7)}$ that if a body is large compared with the scale of turbulence, the flow resistance will increase as compared with non-turbulent flow, and if, on the other hand, the body is smaller than the smallest eddy, it will follow all the turbulence components of the fluid. From this experiment it is concluded that in turbulent liquid flow of tubulence intensity 0.13 and of an integral scale of the same order of magnitude as bubble diameter, the bubble relative velocity shows almost the same value as in quiescent liquids, although shape is very much affected by the turbulence.

\section{Conclusions}

By using a countercurrent flow apparatus, single air bubbles were fixed at a position of the test section by downward flow. Two series of experiments, the undisturbed and the disturbed by an immersed cylinder, were conducted. Bubble relative velocity and shape in the undisturbed experiments were similar to those in quiescent liquids. In the disturbed experiments, in which the intensity of liquid velocity fluctuation was 0.13 and the integral scale was of the same order as the bubble diameter, the bubble relative velocity was substantially the same as in quiescent water, although the shape was changed from time to time by the effect of turbulence. The ratio of the wake region volume to the bubble volume was similar in the two cases, and the averaged value was 4.7.

$$
\begin{aligned}
& \text { Nomenclature } \\
& \text { a } \quad=\text { major axis of bubble } \quad[\mathrm{cm}] \\
& b \quad=\text { minor axis of bubble } \quad[\mathrm{cm}] \\
& C_{D} \quad=\text { drag coefficient }\left(=4 d g / 3 U^{2}\right) \quad[-] \\
& d \quad=\text { equivalent spherical diameter of bubble } \quad[\mathrm{cm}] \\
& f \quad=\text { frequency }[\mathrm{Hz}] \\
& F(f) \quad=\text { one-dimensional energy spectrum function } \\
& \left(=E(f) / \overline{u^{\prime 2}}, \overline{u^{\prime 2}}=\int_{0}^{\infty} E(f) d f\right) \quad \text { [sec] } \\
& R \quad=\text { radius of the test section } \quad[\mathrm{cm}] \\
& \text { Re } \quad=\text { Reynolds number }(=d U / \nu) \text { [-] } \\
& r \quad=\text { radial distance measured from the axis [cm] }
\end{aligned}
$$


$=$ bubble relative velocity or liquid velocity

$u^{\prime} \quad=$ liquid velocity fluctuation

$V_{B} \quad=$ volume of single bubble

$V_{W} \quad=$ volume of wake region

$x=$ distance from the immersed cylinder

$\Lambda_{f}$

$=$ integral scale of turbulence
5) Davidson, J. F. and F. A. Kirk: Chem. Eng. Sci., 24, 1529 (1969).

6) Harberman, W. L. and R. K. Morton: Trans. Am. Soc. Civil Engrs., 121, 227 (1956).

7) Hinze, J. O.: "Turbulence", McGraw-Hill, New York (1959).

8) Kubota, M., T. Akehata and T. Shirai: Kagaku Kogaku, 31, 1074 (1967).

9) Moo-Young, M., G. Fulford and I. Cheyne: Ind. Eng. Chem., Fundamentals, 10, 157 (1971).

10) Subramanian, G. and C. Tien: Preprint of 63rd Annual Meeting of AIChE, I-43d, Chicago (1970).

11) Tadaki, T. and S. Maeda: Kagaku Kögaku, 25, 254 (1961).

12) Taneda, S.: J. Phys. Soc. Japan, 11, 1104 (1956).

13) Uno, S. and R. C. Kintner: AIChE J., 2, 3 (1956).

\title{
CORRELATION OF DATA ON THE APPARENT FRIC- TION COEFFICIENT IN UPWARD TWO-PHASE FLOW OF AIR-LIQUID MIXTURES
}

\author{
TAHEI TOMIDA*, TSUKASA YAMAMOTO, \\ TAMOTSU TAKEBAYASHI AND TATSUYA OKAZAKI \\ Department of Chemical Engineering, Tokushima University, \\ Tokushima, 770
}

\begin{abstract}
Experiments were made on the two-phase pressure drop in vertical upward flow. A similar type of correlation for the apparent friction coefficient to that proposed by Rippel et al. was developed to include the physical properties of liquids. In the correlation the apparent friction coefficient is expressed as a function of the ratio of the mass flow rate of gas and liquid and the liquid Reynolds number. It was found that the change in the slope of the correlation curve corresponded to the transition point of the flow pattern. This type of correlation was applied to data from the literature obtained with various geometries of flow. A correlation between the apparent friction coefficient and liquid film thickness, including wave height, was also proposed.
\end{abstract}

\section{Introduction}

There have been many experimental studies of pressure drops in flow of air-liquid mixtures in vertical or horizontal tubes. Most of the correlations presented are independent of flow pattern changes over a wide range of flow conditions $\mathbf{s}^{1,5,7,11,17,18,27)}$.

Rippel et al. ${ }^{15)}$ proposed a correlation between the pressure drop and the volumetric fraction of liquid input, applying the drag coefficient concept. This correlation is simple and is probably applicable to a wide variety of flow conditions, corresponding to the flow patterns of slug, froth and annular flow regime. But the functional dependence of this correlation on the physical properties of a liquid have not been studied.

Received June 6, 1974.
In this paper, a similar type of correlation to that of Rippel et al. ${ }^{15)}$ is proposed and adapted to more viscous liquid flow and to an extensive flow region. The correlation is applied to other data reported for vertical tubes ${ }^{5,21,22)}$, vertical annular tubes ${ }^{8)}$, vertical rectangular section ${ }^{13)}$, coiled tube ${ }^{15)}$ and horizontal tubes $^{9,11,14,20)}$.

The relation between the apparent friction coefficient and the liquid film thickness, including the wave height, is also examined.

\section{Experimental Apparatus and Procedure}

The equipment used was essentially the same as that described previously ${ }^{23)}$. The test tube used was made of clear acrylate plastics and Pyrex glass, and was mounted vertically in an annular slot-type injector. Table 1 lists the ranges of variables used in 